\title{
Modeling of the formation and transport of nanoparticles in silane plasmas
}

\author{
Kathleen De Bleecker, ${ }^{1, *}$ Annemie Bogaerts, ${ }^{1}$ and Wim Goedheer ${ }^{2}$ \\ ${ }^{1}$ Department of Chemistry, University of Antwerp, Universiteitsplein 1, 2610 Wilrijk, Belgium \\ ${ }^{2}$ FOM-Institute for Plasma Physics "Rijnhuizen," P.O. Box 1207, 3430 BE Nieuwegein, The Netherlands
}

(Received 12 May 2004; revised manuscript received 23 August 2004; published 18 November 2004)

\begin{abstract}
The behavior of nanoparticles in a low-pressure silane discharge is studied with the use of a self-consistent one-dimensional fluid model. Nanoparticles of a given (prescribed) radius are formed in the discharge by the incorporation of a dust growth mechanism, i.e., by including a step in which large anions (typically $\mathrm{Si}_{12} \mathrm{H}_{25}^{-}$), produced in successive chemical reactions of anions with silane molecules, are transformed into particles. Typically a few thousand anions are used for one nanoparticle. The resulting particle density and the charge on the particles are calculated with an iterative method. While the spatial distribution and the charge of the particles are influenced by the plasma, the presence of the nanoparticles will in turn influence the plasma properties. Several simulations with different particle radii are performed. The resulting density profile of the dust will greatly depend on the particle size, as it reacts to the shift of the balance of the different forces acting on the particles.
\end{abstract}

DOI: 10.1103/PhysRevE.70.056407

PACS number(s): 52.27.Lw

\section{INTRODUCTION}

Radiofrequency powered plasma enhanced chemical vapor deposition (PECVD) is often used to deposit hydrogenated amorphous silicon $(a-\mathrm{Si}: \mathrm{H})$ thin films, which are applied in solar cell technology and for the manufacturing of flat panel displays $[1,2]$. When changing plasma conditions, in order to achieve a higher deposition rate, dust formation in the gas phase is observed. Management and avoidance of the formation of these particles in the so-called dusty plasmas was initially the main concern. In the microelectronics industry, for example, the occurrence of dust particles in plasma devices can cause irremediable defects [3], as the feature size of the integrated circuitry elements is rapidly decreasing. More recently, however, it has been shown that the production process of solar cells could in fact benefit from the incorporation of nanocrystalline silicon particles in the $a$ $\mathrm{Si}$ :H matrix [4]. Deposition conditions close to powder formation result in a material with improved properties, as both an increase in lifetime and efficiency is observed $[5,6]$. These so-called polymorphous silicon films prove to be a new potential candidate for the production of high efficiency solar cells. Hence, the formation and growth of nanoparticles is a critical issue in many industrial applications. Therefore, it is essential to understand the growth, charging and transport of these particles in order to minimize or eliminate undesirable contaminants in the semiconductor technology, but also to create new ways for generating desirable, plasma-produced particles for enhanced film properties. Up to now, only a few experimental diagnostics are nonintrusive and especially small dust particles in the initial stage of particle formation are difficult to detect, as the particles can still be below the detection limit of the applied diagnostic. Therefore, a modeling approach can assist to elucidate the reaction mechanisms and the processes occurring in the gas phase.

\footnotetext{
*Electronic address: kathleen.debleecker@ua.ac.be
}

In this paper we report on a study of the formation and behavior of nanoparticles in a silane discharge, by means of numerical simulations with a one-dimensional (1D) fluid model. In our previous growth studies $[7,8]$ we have already confirmed that the negative ions clearly play an important role in the dust formation, as successive reactions of anions with silane appear to be the main growth mechanism. Hence, particle formation and growth are often linked to the presence of negative ions in the rf discharge, which have long residence times in the plasma, as they are electrically confined by the plasma sheaths. So far we have mainly focused on the nucleation phase, i.e. the initial step of particle formation, and have therefore incorporated anions containing up to a maximum of twelve silicon atoms in our model $\left(\mathrm{Si}_{n} \mathrm{H}_{m}^{-}\right.$ with $n \leqslant 12$ ).

Here we address the formation of sub-micrometer particles (ranging in size from 10 to $100 \mathrm{~nm}$ diameter) and their influence on the discharge. In existing models, investigations have so far focused on the charging of dust particles [9-11], transport phenomena (including the forces acting on the particles) [12,13] and plasma-particle interactions [14,15] in undisturbed plasmas. To incorporate another important aspect, i.e., the influence of dust on the discharge, the model has to account self-consistently for the coupling between particle behavior and changes of the plasma properties. Akdim et al. [16] developed a self-consistent model for a dust containing radio frequency discharge, but have not considered the formation mechanisms of the dust. So far none of the performed studies on particle behavior have taken the nucleation or the growth kinetics of the dust particles into account; only separate investigations of particle formation have been performed $[17,18]$. An important extension compared to the model of Akdim et al. is that we have made a coupling between the nucleation of small dust particles (typically up to $\mathrm{Si}_{12} \mathrm{H}_{25}^{-}$) and the formation of nanoparticles with a given prescribed radius. Thus, the nanoparticles simulated in the model are not simply injected from outside the plasma, but are gradually grown due to a series of chemical reactions in the gas phase, better known as gas phase polymerization. 
The presence of dust particles in a plasma can greatly modify its properties and the particles generally acquire a charge (typically negative). Here the nanometer-sized particles are of special interest, as it has been shown that under certain conditions small nanoparticles can somehow escape from the discharge and be deposited into the growing film [19]. In particular, we will focus on the formation of nanoparticles with certain radii and look at some fundamental aspects, such as their specific density profile, their charge, and the influence of the different forces acting on the particles. In a last paragraph we also look at the temperature dependence of the nanoparticle growth.

First, a general overview of the model is given in Sec. II. Besides the description of the fluid model, the behavior of the dust particles in the plasma is described, with emphasis on the charging mechanism, and the various forces acting on the dust particles. The coupling mechanism of the nucleation and the nanoparticle formation is also briefly surveyed. The simulation results of the nanoparticles are presented in Sec. III, followed by the conclusions in Sec. IV.

\section{MODEL DESCRIPTION}

\section{A. General overview of the fluid model}

In order to describe the nucleation of small dust particles and the processes occurring in the reactive silane plasma, a large amount of chemical reactions and species have to be introduced in the model. Under these circumstances, the fluid model is generally considered to be the best approach. We do not reach very low pressures, where the mean free path becomes comparable to the electrode separation and DSMC methods are required. The charging of the dust is a stochastic process that does require a kinetic approach to study its details. This is however beyond the scope of this paper, where chemistry is the main issue.

In the 1D fluid model, applied in this study, the particle balances, the electron energy balance and the Poisson equation are solved numerically on a uniform mesh containing 128 grid points. The electric field is calculated from the Poisson equation, which is coupled to the balance equations, making the model fully self-consistent. For every species $j$ (electrons, ions, radicals, molecules, and nanoparticles) a density balance equation is taken into account. The momentum balance equation is in this case replaced by the driftdiffusion approximation [20] and consists of two separate terms, a drift and a diffusion term. Since the ions can not follow the instantaneous electric field, an effective electric field is used. By adopting this effective electric field the inertia effects of the ions are taken into account. For the neutral species (radicals and molecules), the flux equation reduces to the diffusion term only, as the neutral species do not feel the influence of the electric field. For the nanoparticles, the flux equation becomes more complicated, because these species are also affected by other forces (see below, Sec. II D). Finally, an energy balance is included for the electrons. The electron energy density (i.e. the product of the electron density and the average electron energy) is calculated selfconsistently from the second moment of the Boltzmann equation. The loss of electron energy due to electron impact collisions, including ionization, dissociation, excitation, attachment, and recombination of electrons with ions on the nanoparticle's surface is included. For every electron-neutral reaction, the reaction rate coefficient is computed as a function of the average electron energy by calculating the electron energy distribution function (EEDF) using the Boltzmann equation in a two-term approximation. No energy balance is included for the ions, the neutrals and the nanoparticles. The energy dissipated by the ions is only accounted for in the overall energy balance of the discharge, where a preset total consumed power is specified. A more elaborate description of the different fluid equations can be found in Ref. [20].

For the discretization of the balance equations the Scharfetter-Gummel exponential scheme is applied. The time step within an rf cycle $(50 \mathrm{MHz})$ is set to $2.5 \times 10^{-10} \mathrm{~s}$ (i.e., the rf cycle is divided in 80 time steps), in order to avoid numerical instabilities. Convergence of the fluid model is reached when the relative changes of the discharge parameters between two succeeding rf cycles are less than $10^{-6}$. For more information concerning the applied numerical techniques and algorithms we refer to Ref. [21].

The same fluid model for a 'standard' silane plasma (i.e., without the inclusion of dust formation) has already been extensively tested with experimental results and good agreement between modeling and experimental results was obtained [20].

\section{B. Implementing nanoparticles in the fluid model}

The anionic pathway is generally believed to be the most viable route for the generation of dust particles in silane plasmas [22-24], as the anions are electrostatically trapped by the ambipolar potential in the plasma bulk. The long residence time of the negative ions in the plasma, favours their further growth, and makes them good candidates to trigger particle nucleation. Consequently, they can easily undergo polymerization reactions, which will ultimately lead to the formation of nanometer and micrometer sized particles. Furthermore, mass spectra, measured by Hollenstein et al. [25], show a good correlation between the anions and particle formation.

In our model dust is primarily formed by successive reactions of anions with silane molecules (ground state and vibrationally excited silane $\mathrm{SiH}_{4}^{(2-4)}$ and $\mathrm{SiH}_{4}^{(1-3)}$ molecules) [8]. The earliest stages of particle formation start primarily from the anions $\mathrm{SiH}_{3}^{-}$and $\mathrm{SiH}_{2}^{-}$, principally formed through the electron induced dissociative attachment of $\mathrm{SiH}_{4}$,

$$
\mathrm{SiH}_{4}+e^{-} \rightarrow \mathrm{SiH}_{x}^{-}+\mathrm{H}_{4-x} \quad(x=2 \text { or } 3)
$$

with over $70 \%$ leading to the formation of $\mathrm{SiH}_{3}^{-}$.

Two different pathways, starting from either $\mathrm{SiH}_{3}^{-}$or $\mathrm{SiH}_{2}^{-}$, can be distinguished and lead to the growth of larger silyl $\left(\mathrm{Si}_{n} \mathrm{H}_{2 n+1}^{-}\right)$and silylene $\left(\mathrm{Si}_{n} \mathrm{H}_{2 n}^{-}\right)$anions, respectively, due to successive anion- $\mathrm{SiH}_{4}$ polymerization reactions,

$$
\mathrm{Si}_{n} \mathrm{H}_{2 n+1}^{-}+\mathrm{SiH}_{4} \rightarrow \mathrm{Si}_{n+1} \mathrm{H}_{2 n+3}^{-}+\mathrm{H}_{2}
$$




$$
\mathrm{Si}_{n} \mathrm{H}_{2 n}^{-}+\mathrm{SiH}_{4} \rightarrow \mathrm{Si}_{n+1} \mathrm{H}_{2 n+2}^{-}+\mathrm{H}_{2}
$$

at a rate constant of the order of $10^{-18} \mathrm{~m}^{3} \mathrm{~s}^{-1}$. In addition to ground state silane molecules, reactions with vibrationally excited silane molecules, formed by electron impact on ground state $\mathrm{SiH}_{4}$, i.e. $\mathrm{SiH}_{4}^{(2-4)}$, at $0.113 \mathrm{eV}$, and $\mathrm{SiH}_{4}^{(1-3)}$, at $0.271 \mathrm{eV}$, are also considered in the fluid model. These reactions can in fact enhance the kinetics of the clustering mechanism [8].

Since it is not possible to describe in detail the plasma chemistry for an unlimited number of plasma species, both pathways are stopped in the model at anions containing 12 silicon atoms, leading to the formation of $\mathrm{Si}_{12} \mathrm{H}_{25}^{-}$and $\mathrm{Si}_{12} \mathrm{H}_{24}^{-}$, respectively. In order to simulate the further nanoparticle's growth, we have introduced a step which immediately transforms these large anions (mostly $\mathrm{Si}_{12} \mathrm{H}_{25}^{-}$), into nanoparticles of a given radius. Hence, instead of introducing an anion-silane reaction for $\mathrm{Si}_{12} \mathrm{H}_{25}^{-}$and $\mathrm{Si}_{12} \mathrm{H}_{24}^{-}$which would lead to the formation of silicon hydride anions containing 13 silicon atoms (e.g., $\mathrm{Si}_{12} \mathrm{H}_{25}^{-}+\mathrm{SiH}_{4} \rightarrow \mathrm{Si}_{13} \mathrm{H}_{27}^{-}+\mathrm{H}_{2}$ at $k=10^{-18} \mathrm{~m}^{3} \mathrm{~s}^{-1}$ ), we have used the production rate of these species as the source term for the formation of nanoparticles with a certain prescribed radius. As will be shown in Sec. III, several simulations are performed where the nanoparticle radius is varied between 10 and $100 \mathrm{~nm}$. Hence, for every simulation the radius of the nanoparticles is given, whereas their charge and density and flux are calculated with an iterative method (see below).

\section{Charging of the nanoparticles}

Nanoparticles immersed in a plasma become charged by collecting plasma ions and electrons. In absence of any radiative environment, the particles typically acquire a negative equilibrium charge, as the thermal speed of the electrons by far exceeds that of the positive ions. The magnitude of the negative charge on a nanoparticle will strongly depend on the particle size and the plasma conditions. This negative charge will also be responsible for the confinement and consequently longer lifetimes of such particles in the plasma.

The (average) charge on a nanoparticle is obtained from the balance between the electron and positive ion currents towards the particle's surface. The anions, although abundantly present in the plasma, do not contribute to the current balance, as the negative ions do not have enough kinetic energy to overcome the negative floating potential of the dust particle. For a spherical nanoparticle with radius $r_{d}$, the Orbital-Motion-Limited (OML) probe theory [26] can be used to describe the charging currents. The OML theory is only valid if $\mathrm{r}_{d} \ll \lambda_{L}$, where $\lambda_{L}=\left[\left(1 / \lambda_{e}\right)^{2}+\left(1 / \lambda_{i}\right)^{2}\right]^{-1 / 2}$ is the so-called linearized Debye length, which is a combination of the electron Debye length, $\lambda_{e}=\left(\epsilon_{o} k_{B} T_{e} / n_{e} e^{2}\right)^{1 / 2}$, and the ion Debye length, $\lambda_{i}$.

Assuming Maxwellian energy distributions for electrons and ions, the positive ion and electron currents hitting the particles can be described by [26]

$$
I_{i}=4 \pi r_{d}^{2} e n_{i} \sqrt{\frac{k_{B} T_{i}}{2 \pi m_{i}}}\left(1-\frac{e V_{f l}}{k_{B} T_{i}}\right)
$$

and

$$
I_{e}=4 \pi r_{d}^{2} e n_{e} \sqrt{\frac{k_{B} T_{e}}{2 \pi m_{e}}} \exp \left(\frac{e V_{f l}}{k_{B} T_{e}}\right)
$$

where $n_{e(i)}$ is the electron (positive ion) density, $e$ the elementary charge, $k_{B}$ the Boltzmann constant, $T_{e(i)}$ the electron (positive ion) temperature, $m_{i}$ the ion mass, $m_{e}$ the electron mass, and $V_{f l}$ the floating potential (acquired by the nanoparticle relative to the surrounding plasma). The floating potential of the nanoparticle is assumed to be constant during an rf cycle, as the currents towards the nanoparticle's surface are too small to change the charge significantly during an $\mathrm{rf}$ cycle [16]. In the plasma sheaths near the electrodes the ions obtain a drift velocity $v_{i}$ due to the electric field. Therefore, $k_{B} T_{i}$ in Eq. (3) is replaced in the model by the mean energy $E_{i}$; see Ref. [16].

By equating $I_{e}=I_{i}$ [Eqs. (3) and (4)], the floating potential, $V_{f l}$, and consequently the equilibrium charge $Q_{d}=4 \pi \epsilon_{0} r_{d} V_{f l}$ on the nanoparticle can be found. Hence, it seems that the charge on a spherical nanoparticle is proportional to its radius.

The additional complication of stochastic fluctuations superimposed on the equilibrium charge of the nanoparticle, is not taken into account. These fluctuations are a consequence of the random nature of the collection of electrons and ions on the nanoparticle's surface (collection occurs at random times and sequences). This effect will, however, be reviewed at a later stage.

Note, however, that the recombination of ions and electrons on the dust particle's surface is taken into account, by means of a recombination rate [16], and represents an additional important loss mechanism for the electrons and ions residing in the plasma.

\section{Forces acting on the nanoparticles}

Once we have obtained the charge on the nanoparticle's surface, we are able to calculate the electrostatic force exerted by the electric field $E$ on the particle. Daugherty et al. [27] derived the following expression,

$$
F_{e}=Q_{d} E\left(1+\frac{\left(r_{d} / \lambda_{L}\right)^{2}}{3\left(1+r_{d} / \lambda_{L}\right)}\right)
$$

where $Q_{d}$ is the charge on the nanoparticle, $E$ is the electric field and $\lambda_{L}$ is the linearized Debye length. Generally, $\mathrm{r}_{d}$ $\ll \lambda_{L}$, making the expression between brackets approximately equal to 1 and the electric force on the particle is then given by

$$
F_{e}=Q_{d} E
$$

This electric force will be responsible for the confinement of negatively charged dust particles in the discharge region and is usually a dominant force for small nanoparticles.

A second force, which can significantly affect the dust particle's transport is due to gravitation. For a spherical particle the gravitational force can be written as 


$$
F_{g}=\frac{4}{3} \pi r_{d}^{3} \rho_{d} g
$$

where $r_{d}$ is the nanoparticle's radius, $\rho_{d}$ is the mass density and $g$ the gravitational acceleration. For amorphous silicon $\rho_{d}$ is about $2.1 \times 10^{3} \mathrm{~kg} / \mathrm{m}^{3}$ [16]. The gravitational force is proportional to the particle mass. In the case of submicrometer particles, the gravitation can be neglected, as the influence of other forces, such as the electrostatic force, are much stronger for particles smaller than a few micrometers.

A third force, the neutral drag force, results from collisions with neutral gas molecules and causes a momentum transfer from the neutral gas to the nanoparticles. Using the kinetic gas theory, the neutral drag force can be approximated by [28]

$$
F_{n}=-\frac{4}{3} \pi r_{d}^{2} n_{n} m_{n} v_{t h}\left(v_{d}-v_{n}\right),
$$

where $n_{n}$ is the neutral gas number density, $m_{n}$ is the mass of a neutral molecule, $v_{n}$ the velocity of the gas, $v_{d}$ the drift velocity of the particle, and $v_{t h}$ the average thermal velocity of the gas. Advection of the gas is not included in the present model $\left(v_{n}=0\right)$, which means that the neutral drag force will only act as a damping force on the velocity of the nanoparticles.

A second important drag force that acts on the nanoparticles is the ion drag force. It is caused by the momentum transfer from the positive ions to the nanoparticles driven by the electric field. This force consists of two components: the collection and the orbit force. The collection force represents the momentum transfer of all the ions that are collected by the particle. Each impinging ion transfers its original momentum, $m_{i} v_{i}$, making the collection force equal to [12]

$$
F_{i}^{c}=\pi b_{c}^{2} n_{i} v_{s} m_{i} v_{i}
$$

where $v_{s}$ is the mean speed of the positive ions and $b_{c}$ the collection parameter.

The second component, the orbit force, is given by [12]

$$
F_{i}^{o}=4 \pi b_{\pi / 2}^{2} \Gamma n_{i} v_{s} m_{i} v_{i}
$$

where $b_{\pi / 2}$ is the impact parameter that corresponds to the deflection angle $\pi / 2$ and

$$
\Gamma=\frac{1}{2} \ln \left(\frac{\lambda_{L}^{2}+b_{\pi / 2}^{2}}{b_{c}^{2}+b_{\pi / 2}^{2}}\right)
$$

is the Coulomb logarithm. The orbit force is caused by positive ions that do not reach the particle's surface, but transfer their momentum by Coulomb interactions.

Finally, the thermophoretic force can be induced by the existence of a gas temperature gradient in the discharge (e.g., due to heating/cooling of the electrodes). More momentum is transferred from gas molecules in the hotter area to the nanoparticles in comparison to gas molecules in colder regions of the plasma. Hence, the nanoparticles experience a net force in the direction of $-\nabla T_{\text {gas }}$. The thermophoretic force is given by [29]

$$
F_{t h}=-\frac{32}{15} \frac{r_{d}^{2}}{v_{t h}}\left(1+\frac{5 \pi}{32}(1-\alpha)\right) \kappa_{T} \frac{d T_{\text {gas }}}{d x}
$$

with $v_{t h}$ the average thermal velocity of the gas. The thermal accommodation coefficient, $\alpha$, is taken equal to 1 and $\kappa_{T}$ represents the translation part of the thermal conductivity.

The expression for the flux of the nanoparticles is obtained by assuming that the neutral drag force, which is considered here as a friction force, balances the sum of all other forces. Using the expression of the momentum loss frequency,

$$
\nu_{m d}=\sqrt{2} \frac{p_{t o t}}{k_{B} T_{g a s}} \pi r_{d}^{2} \sqrt{\frac{8 k_{B} T_{g a s}}{\pi m_{d}}}
$$

and the mobility and diffusion coefficient,

$$
\begin{gathered}
\mu_{d}=\frac{Q_{d}}{m_{d} \nu_{m d}}, \\
D_{d}=\mu_{d} \frac{k_{B} T_{g a s}}{Q_{d}}
\end{gathered}
$$

a drift-diffusion expression for the flux of the nanoparticles is obtained [16],

$$
\begin{aligned}
\Gamma_{d}= & -\mu_{d} n_{d} E_{e f f}-D_{d} \frac{d n_{d}}{d x}-\frac{n_{d}}{\nu_{m d}} g+\sum \frac{n_{d} m_{i} v_{s}}{m_{d} \nu_{m d}} \\
& \times\left(4 \pi b_{\pi / 2}^{2} \Gamma+\pi b_{c}^{2}\right) \Gamma_{i}-\frac{32}{15} \frac{n_{d} r_{d}^{2}}{m_{d} \nu_{m d} v_{t h}} \kappa_{T} \frac{d T_{g a s}}{d x} .
\end{aligned}
$$

As a result, the nanoparticles can be treated with the same numerical procedures as the other charged species in the model.

As the drift velocity and the diffusion coefficient of the nanoparticles are much smaller than those of the positive and negative ions and the electrons, a different calculation cycle with a different time step is introduced for the computation of the density and the charge of the nanoparticles [16]. Hence, first the transport equations of the ions, electrons and the Poisson equation are solved during a number of rf cycles (i.e., the first calculation cycle), during which the nanoparticles do not move. During the second calculation cycle, the transport equation of the nanoparticles is solved with a greater time step, using the time averaged electric field, and electron and positive ion fluxes. This causes, however, the creation of space charge regions, as the electron and positive ion densities do not change. These space charge regions will lead to instabilities in the solution of the Poisson equation and the electron transport when switching back to the first calculation cycle. Therefore, the artificially generated space charge regions are corrected by adapting the positive ion density distributions prior to the next series of rf cycles, in which the nanoparticles are stationary and the ion and the electron density profiles adapt themselves to newly calculated density profile of the nanoparticles. By adopting this method a smaller computational effort is required. 


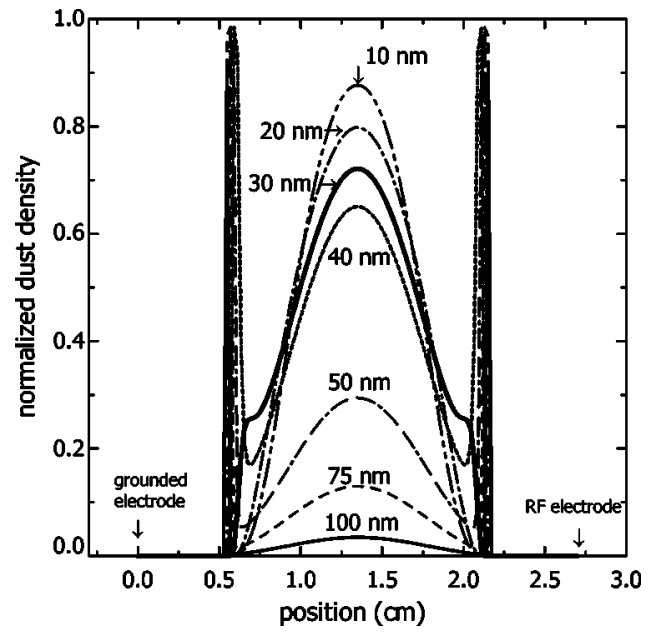

FIG. 1. Computed normalized nanoparticle densities as a function of position in the plasma. The curves of 50,75, and $100 \mathrm{~nm}$ are divided by a factor of 2,4 , and 10 , respectively, in order to fit all the data on the figure.

\section{RESULTS AND DISCUSSION}

In this section the results obtained with the 1D fluid model are presented. The simulated silane discharge is characterized by two parallel plates separated by a gap of $2.7 \mathrm{~cm}$, at a pressure of $40 \mathrm{~Pa}$, a power of $5 \mathrm{~W}$, a driving frequency of $50 \mathrm{MHz}$, and a gas temperature of $400 \mathrm{~K}$. No heating or cooling of the electrodes is incorporated in the present model, hence the thermophoretic force is neglected. In all calculations the input gas flow of silane is set at $20 \mathrm{sccm}$. The only parameter that is varied is the size of the nanoparticles (between 10 and $100 \mathrm{~nm}$ in diameter). Only in the last simulation the gas temperature is varied between $293 \mathrm{~K}$ and $500 \mathrm{~K}$ in order to be able to investigate the temperature dependence of the nanoparticle growth. The result is shown at the end of this section for particles of $10 \mathrm{~nm}$ in size.

\section{A. Density profiles of the nanoparticles}

Figure 1 shows the calculated normalized density profiles of the nanoparticles, at the conditions discussed above. Each plot represents a separate simulation, where the production rate of species with more than 12 silicon atoms leads to the formation of particles of $10,20,30,40,50,75$ or $100 \mathrm{~nm}$, respectively. In the present simulations no steady state can be achieved, as the nanoparticles continuously build up in time. Obviously, at any given time the growth of larger nanoparticles will be less extensive compared to that of smaller nanoparticles, therefore normalized density profiles are adopted. Figure 1 clearly shows that the resulting density profile greatly depends on the nanoparticle's size, as it reacts to the shift of the balance of the different forces acting on the particles. For nanoparticles up to $30 \mathrm{~nm}$, the density profile exhibits a maximum in the bulk of the discharge between the two electrodes, whereas the profile of larger nanoparticles tends to move towards the boundaries of the plasma, thereby diminishing its concentration in the center of the discharge. Hence, the competition between the different forces results

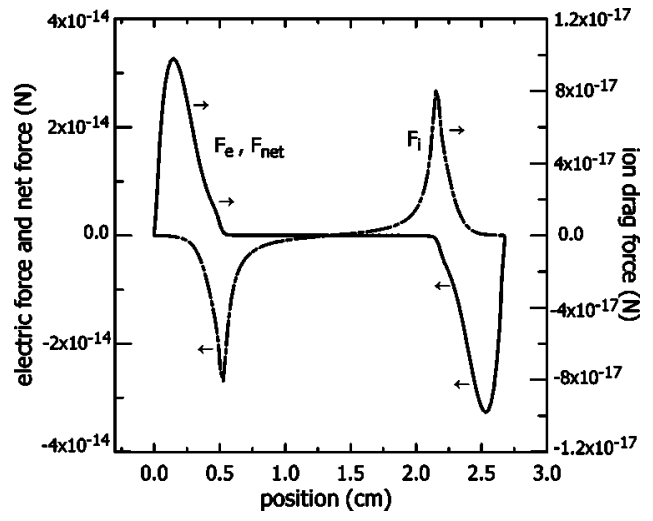

FIG. 2. Calculated electric force $\left(F_{e}\right)$, ion drag $\left(F_{i}\right)$, and net force $\left(F_{\text {net }}\right)$ acting on nanoparticles of $10 \mathrm{~nm}$. The electric and net force are indicated by a solid line; and the ion drag force by a dashed line. The direction of the forces is indicated by left and right arrows.

in trapping of the nanoparticles in some well defined regions of the discharge.

\section{B. The different forces acting on the nanoparticles}

Since no thermophoretic force is applied and the force due to gravity is negligible for such small particles, the nanoparticles of a certain size will accumulate where the electrostatic and the ion drag force balance. Indeed, we have assumed that the neutral drag force only acts as a damping force and hence is in equilibrium with the sum of all other forces, which means in practice, that it is equal to the net force resulting from the balance of the electrostatic and the ion drag force. This assumption concurs with the proposal of Barnes et al. [12] and Sommerer et al. [30], who argue that the transport of small particles is actually dominated by two forces, the electrostatic and the ion drag force. The electrostatic, the ion drag force and the resulting net force are plotted as a function of position in Fig. 2 for a particle of $10 \mathrm{~nm}$. It is clearly demonstrated that the forces on the particles are very sensitive to the location within the silane discharge. In the sheath regions near the electrodes, where large electric fields are present, the electrostatic force far exceeds the ion drag force. Hence, the electrostatic force will accelerate the negatively charged nanoparticles to the center of the discharge, where the potential is usually most positive, and totally dominates the net force. However, in low electric fields, as in the plasma bulk, the ion drag force can in some cases exceed the electrostatic force, and accelerates the nanoparticles in the direction of the net positive ion flux, which is generally towards the plasma boundaries.

To visualize this effect we need to focus our attention to what happens in the bulk of the discharge. Therefore, only part of the electrostatic, the ion drag force and the resulting net force are presented in Fig. 3, i.e. the part between 0.5 and $2.2 \mathrm{~cm}$. We have calculated the forces for 4 specific cases, namely for particles of $10,30,50$, and $100 \mathrm{~nm}$, repectively. For comparison, the normalized density profile is also represented in each plot by a thin dotted line. Each of the forces has a different power-law dependency on the particle radius 

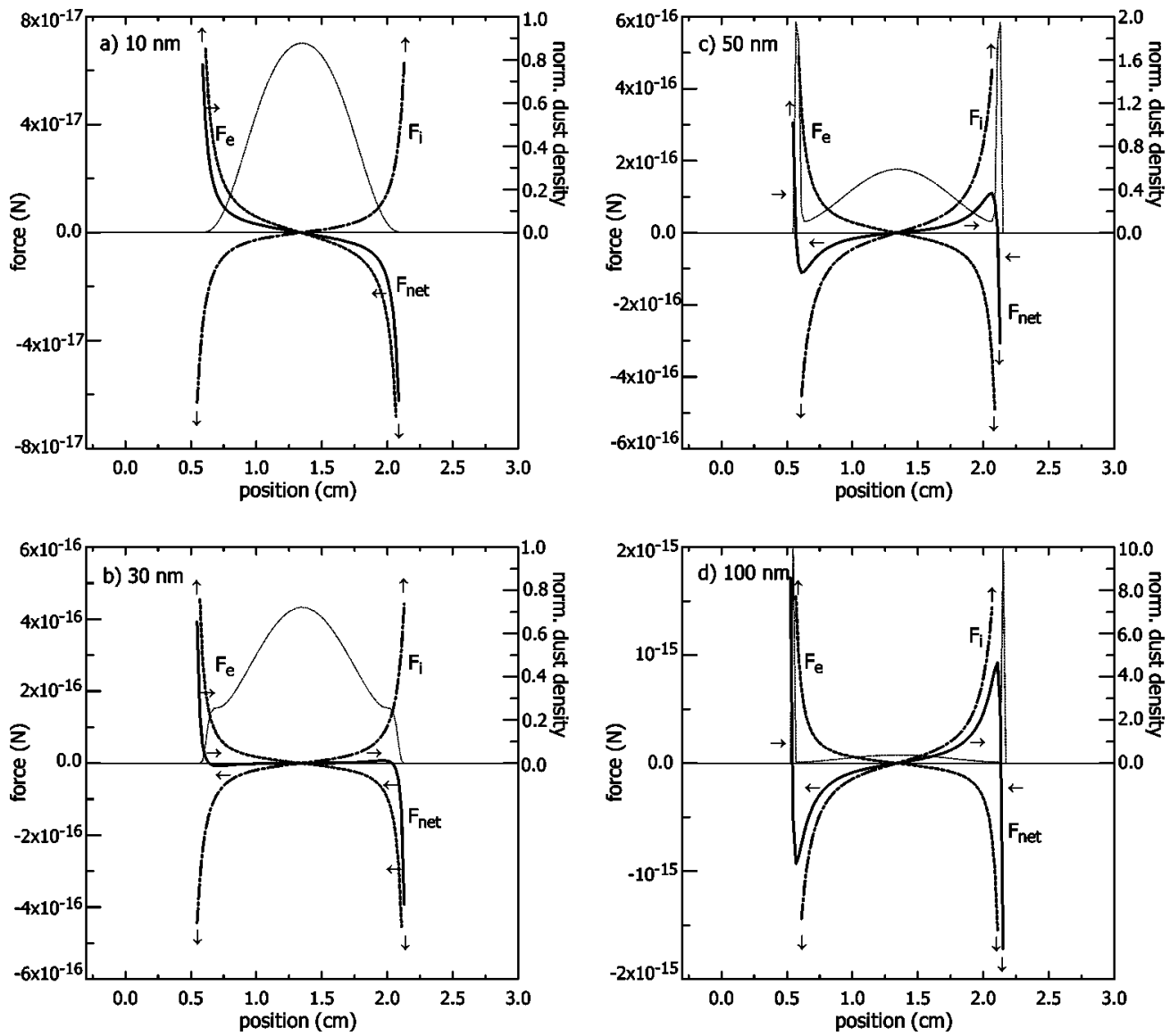

FIG. 3. Calculated electric force $\left(F_{e}\right)$, ion drag force $\left(F_{i}\right)$, and net force $\left(F_{n e t}\right)$ acting on nanoparticles of (a) $10 \mathrm{~nm}$; (b) $30 \mathrm{~nm}$; (c) $50 \mathrm{~nm}$; and (d) $100 \mathrm{~nm}$. The forces are only shown in the bulk region of the discharge, i.e., between 0.5 and $2.2 \mathrm{~cm}$. The direction of the net force, represented by a solid line, is indicated by left and right arrows. The normalized density profile is also shown: thin dotted line, right axis.

(see equations Sec. II D), meaning that the dominant force will change as the particle becomes larger in size.

For particles of $10 \mathrm{~nm}$ [Fig. 3(a)], it appears that the electrostatic force remains the most dominant force even in the center of the discharge. Hence, the particles are forced to the center of the plasma, where the particle density profile exhibits a local maximum (see thin dotted line).

For 50 and $100 \mathrm{~nm}$ particles [Fig. 3(c) and Fig. 3(d)], the ion drag force is the most dominant force in the center of the discharge and moves the particles towards the electrodes until they begin to feel the electric field at approximately 0.6 and $2.1 \mathrm{~cm}$ for a $50 \mathrm{~nm}$ particle, and 0.5 and $2.2 \mathrm{~cm}$ for a $100 \mathrm{~nm}$ particle, respectively. At this point the electrostatic and the ion drag forces balance each other and the density profile shows a pronounced maximum [see peaks in Figs. 3(c) and 3(d)].

The density profile of $30 \mathrm{~nm}$ particles exhibits some peculiar behavior due to the action of the different forces [Fig. 3(b)]: although the electric force still forces the particles to the center of the discharge, where a local maximum is attained(see thin dotted line), the particles also begin to feel the ion drag force, which results in this specific density profile with shoulders near the sheaths.

In conclusion, it is clear that the ion drag force scales more strongly with the radius of the nanoparticles in comparison to the electric force. Therefore, for particles larger than $30 \mathrm{~nm}$ the forces balance further to the boundary of the plasma, thereby trapping the particles in some well defined regions near the sheaths, whereas in a similar fashion particles smaller than $30 \mathrm{~nm}$ are forced to the center of the discharge. So, under the present conditions it seems that the ion drag force only becomes a dominant force for particles which are larger than $30 \mathrm{~nm}$ in size.

\section{Charge on the nanoparticles}

Figure 4 shows the calculated charge collected on the nanoparticle's surface as a function of position in the plasma for various sizes of nanoparticles. The charge distribution for each nanoparticle size is calculated using the OML theory, as was explained above. Every charge profile seems to vary significantly depending on the local electron and ion densities and the electron temperature. Near the electrodes, in the sheaths, few electrons can be found, resulting in a sharp decrease in the nanoparticle's charge profile. In the pre-sheaths the electrons are accelerated by the electric field and they have sufficient energy to overcome the repulsive Coulomb force caused by the similar charge on the nanoparticle's surface. This results in a high charge on the nanoparticles. In the plasma bulk, however, the electrons have more difficulty to overcome the repulsive Coulomb force, due to their lower electron energy. Hence, the charge on the nanoparticle is much lower in the center of the discharge. 


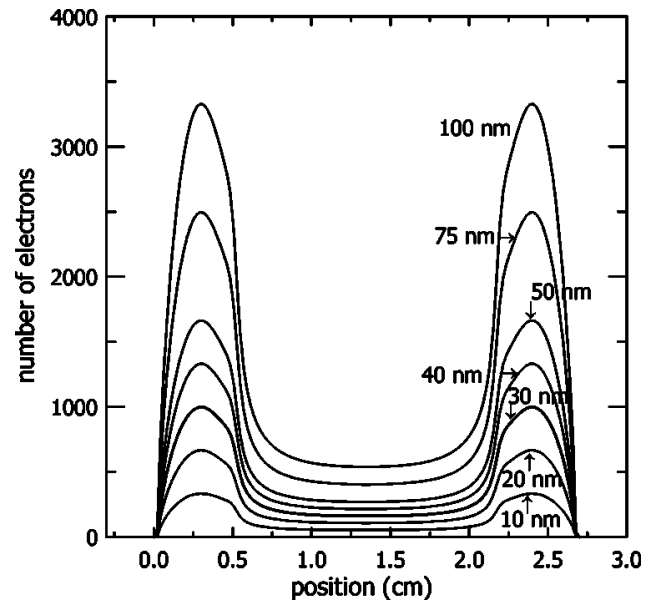

FIG. 4. Calculated charge on nanoparticles of 10 to $100 \mathrm{~nm}$ in number of electrons as function of position in the gas discharge.

Figure 4 evidently suggests that if particles want to escape from the discharge they will have to overcome the maximum negative charge located in the presheath. Note that only neutral and positively charged particles will be able to escapefrom the discharge, by either diffusion or drift, respectively, and can be deposited in the growing film, whereas negative particles are repelled by the electric field. Hence, particles with a small negative charge (in the order of one or two negative charges) can be neutralized or positively charged, but particles with larger negative charges remain trapped in the discharge by the ambipolar potential. Under the present conditions it seems that even particles of $10 \mathrm{~nm}$ will not be able to leave the discharge. It can also be seen that an increase in nanoparticle size results in a higher negative charge on the nanoparticle's surface.

For clarity, Fig. 5 shows the number of electrons per nanoparticle at the position in the discharge where most nanoparticles are present, i.e., where the maximum occurs in their density profile (see Fig. 1). Note that the charge rises dramatically for increasing nanoparticle size: small particles

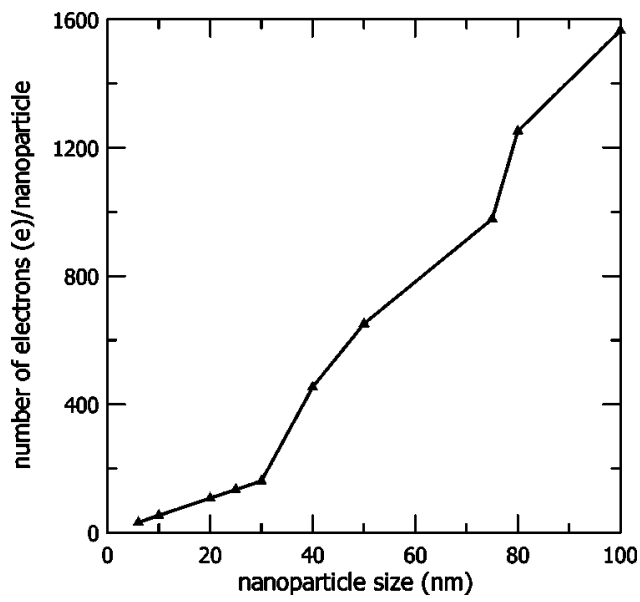

FIG. 5. Computed number of electrons per nanoparticle as a function of nanoparticle size at the position in the discharge where most nanoparticles are present.

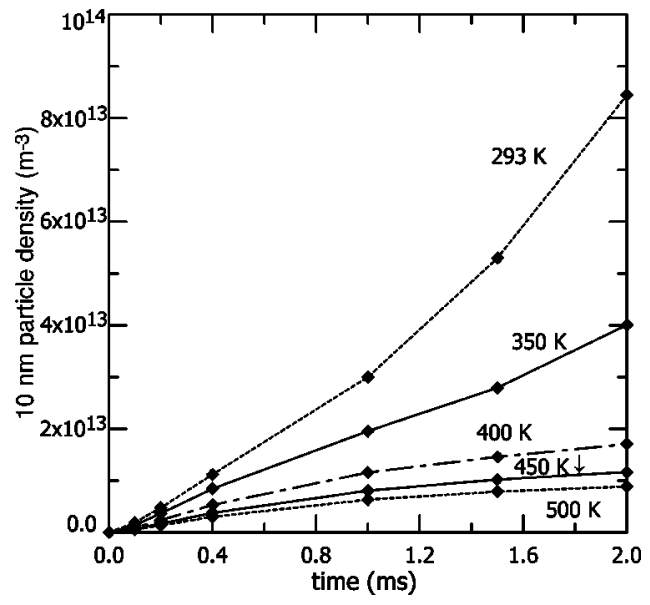

FIG. 6. Calculated evolution of the growth of $10 \mathrm{~nm}$ particles at different gas temperatures.

(e.g., $10 \mathrm{~nm}$ ) have a charge of 32 electrons, whereas most $100 \mathrm{~nm}$ particles can hold as much as 1600 electrons, according to the calculations with the standard OML theory. This result is higher than expected, suggesting that the OML theory maybe overestimates the charge of particles in the nanometer range.

\section{Effect of gas temperature on nanoparticle growth}

In order to investigate the temperature dependence of the nanoparticle's growth, we have performed several simulations at different gas temperatures ranging between $293 \mathrm{~K}$ and $500 \mathrm{~K}$. The results are plotted in Fig. 6 for nanoparticles of $10 \mathrm{~nm}$. The number density represented in the plot refers to that of the center of the discharge, where the density profile attains a local maximum (see Fig. 1). Note that we are interested in the difference of particle growth at different gas temperatures, rather than the absolute values of the number density. Indeed, since we have included a step which immediately transforms particles containing twelve silicon atoms into particles of $10 \mathrm{~nm}$, the growth of the particles is only approximated here and might be faster than the actual growth rate in experimental setups.

To account for the influence of the gas temperature, the mobility and diffusion coefficient of every species is recalculated in our model at each given temperature $T$. Reaction rate constants in the nucleation model (see Ref. [8]) that involve a temperature dependence are also readjusted to the specific temperature $T$, including the deexcitation of vibrational excited silane molecules. Figure 6 clearly shows that the growth of the nanoparticles is affected by the gas temperature: the higher the gas temperature, the lower is the nanoparticle density after a certain time of growth. This means that the growth rate is lower at higher gas temperatures, which corresponds to a delay of the particle growth. This phenomena has also been observed in the experiments of Boufendi et al. [31], where the appearance of visible dust particles is delayed at higher gas temperatures. At room temperature small particles appear almost immediately (i.e., after a few milliseconds), while at $500 \mathrm{~K}$ it will take more than 
$10 \mathrm{~s}$ after ignition of the rf discharge before some dust particles are observed [31].

Several possible explanations for the delay of particle nucleation have been proposed [32]. In our previous model we have already argued that both the temperature dependence of the mobility and diffusion coefficients, as well as the faster vibrational relaxation will probably play an important role [8]. Hence, increasing the gas temperature can in fact be a very effective method for suppressing the dust formation in applications where the nanoparticle formation is not desired.

\section{SUMMARY AND CONCLUSIONS}

In this article we report on the growth and transport of nanoparticles in a low pressure silane discharge. We have used a 1D self-consistent fluid model that couples the particle nucleation mechanisms to the formation and growth of the nanoparticles.

We have investigated the importance of the different forces acting on the nanoparticles and observed that the density profile significantly reacts to a shift of the balance of the different forces acting on the nanoparticles. For particles less than $30 \mathrm{~nm}$ in size, the electrostatic force is the most dominant force, resulting in a density profile with its maximum in the center of the discharge. Larger nanoparticles will experience the ion drag force and are pushed towards the reactor boundaries until the force is balanced by the the force due to the electric field. This leads to the trapping of the nanoparticles in some well defined region of the plasma.

The charge on a nanoparticle has also been calculated, by means of the OML theory. Due to their large negative charge, especially large nanoparticles will remain trapped in the discharge. Fluctuations on the particle's charge (especially important for small nanoparticles) are at this point not taken into account in the model, but this effect will be added in a later stage.

Finally we have shown that the nanoparticle growth experiences an obvious delay when the gas temperature is increased, which concurs with the experimental results obtained by Boufendi et al.

\section{ACKNOWLEDGMENTS}

The authors would like to thank Dr. R. Akdim for his advice and discussions and also for supplying the changes to the fluid code. K.D.B. is indebted to the Institute for the Promotion of Innovation through Science and Technology in Flanders (IWT-Vlaanderen) for financial support. A.B. acknowledges financial support from the Flemish Fund for Scientific research (FWO).
[1] J. Perrin, J. Schmitt, C. Holenstein, A. Howling, and L. Sansonnens, Plasma Phys. Controlled Fusion 42, B353 (2000).

[2] O. Saadame, C. Longeaud, S. Lebib, and P. Roca i Cabarrocas, Thin Solid Films 427, 241 (2003).

[3] G. S. Selwyn, J. Singh, and R. S. Benett, J. Vac. Sci. Technol. A 7, 2758 (1989).

[4] A. Fontcuberta i Morral and P. Roca i Cabarrocas, Thin Solid Films 383, 161 (2001).

[5] M. Meaudre, R. Meaudre, R. Butté, and S. Vignoli, J. Appl. Phys. 86, 946 (1999).

[6] P. Roca i Cabarrocas, J. Non-Cryst. Solids 266-269, 31 (2000).

[7] K. De Bleecker, A. Bogaerts, W. Goedheer, and R. Gijbels, IEEE Trans. Plasma Sci. 32, 691 (2004).

[8] K. De Bleecker, A. Bogaerts, R. Gijbels, and W. Goedheer, Phys. Rev. E 69, 056409 (2004).

[9] J. Goree, Plasma Sources Sci. Technol. 3, 400 (1994).

[10] S. A. Trigger and P. P. J. M. Schram, J. Phys. D 32, 234 (1999).

[11] S. J. Choi and M. J. Kushner, IEEE Trans. Plasma Sci. 22, 138 (1994).

[12] M. S. Barnes, J. H. Keller, J. C. Forster, J. A. O’Neill, and D. K. Coultas, Phys. Rev. Lett. 68, 313 (1992).

[13] J. Perrin, P. Molinàs-Mata, and Ph. Belenguer, J. Phys. D 27, 2499 (1994).

[14] Ph. Belenguer et al., Phys. Rev. A 46, 7923 (1992).

[15] J. P. Boeuf, Phys. Rev. A 46, 7910 (1992).

[16] M. R. Akdim and W. Goedheer, J. Appl. Phys. 94, 104 (2003).

[17] A. Gallagher, A. A. Howling, and Ch. Hollenstein, J. Appl. Phys. 91, 5571 (2002).
[18] U. V. Bhandarkar, M. T. Swihart, S. L. Girshick, and U. R. Kortshagen, J. Phys. D 33, 2731 (2000).

[19] P. Roca i Cabarrocas, P. Gay, and A. Hadjadj, J. Vac. Sci. Technol. A 15, 655 (1996).

[20] G. J. Nienhuis, W. J. Goedheer, E. A. G. Hamers, W. G. J. H. M. van Sark, and J. Bezemer, J. Appl. Phys. 82, 2060 (1997).

[21] J. D. P. Passchier and W. J. Goedheer, J. Appl. Phys. 73, 1073 (1993).

[22] J. Perrin, C. Böhm, R. Etemadi, and A. Lloret, Plasma Sources Sci. Technol. 3, 252 (1994).

[23] A. Gallagher, Phys. Rev. E 62, 2690 (2000).

[24] A. A. Howling, L. Sansonnens, J-L. Dorrier, and Ch. Hollenstein, J. Phys. D 26, 1003 (1993).

[25] Ch. Hollenstein, J-L. Dorier, J. Dutta, L. Sansonnens, and A. A. Howling, Plasma Sources Sci. Technol. 3, 278 (1994).

[26] J. E. Allen, B. M. Annaratone, and U. De Angelis, J. Plasma Phys. 63, 299 (2000).

[27] J. E. Daugherty, R. K. Porteous, and D. B. Graves, J. Appl. Phys. 73, 1617 (1993).

[28] D. B. Graves, J. E. Daugherty, M. D. Kilgore, and R. K. Porteous, Plasma Sources Sci. Technol. 3, 433 (1994).

[29] L. Talbot, R. K. Cheng, R. W. Schefer, and D. R. Willis, J. Fluid Mech. 101, 737 (1980).

[30] T. J. Sommerer, M. S. Barnes, J. H. Keller, M. J. McCaughey, and M. J. Kushner, Appl. Phys. Lett. 59, 638 (1991).

[31] L. Boufendi et al., J. Appl. Phys. 76, 148 (1994).

[32] U. Bhandarkar, U. Kortshagen, and S. L. Girshick, J. Phys. D 36, 1399 (2003). 